\title{
Look Who's Talking: Modeling Decision Making Based on Source Credibility
}

\author{
Andrzej Kawiak ${ }^{1}$, Grzegorz M. Wójcik ${ }^{1(凶)}$, Lukasz Kwasniewicz ${ }^{1}$, \\ Piotr Schneider ${ }^{1}$, and Adam Wierzbicki ${ }^{2}$ \\ 1 Chair of Neuroinformatics and Biomedical Engineering, \\ Maria Curie-Sklodowska University, ul. Akademicka 9, 20-033 Lublin, Poland \\ gmwojcik@live.umcs.edu.pl \\ 2 Polish-Japanese Academy of Information Technology, \\ ul. Koszykowa 86, 02-008 Warsaw, Poland
}

\begin{abstract}
Understanding how humans evaluate credibility is an important scientific question in the era of fake news. Source credibility is among the most important aspects of credibility evaluations. One of the most direct ways to understand source credibility is to use measurements of brain activity of humans who make credibility evaluations. Nevertheless, source credibility has never been investigated using such a method before. This article reports the results of an experiment during which we have measured brain activity during source credibility evaluation using EEG. The experiment allowed for identification of brain areas that were active when a participant made positive or negative source credibility evaluations. Based on experimental data, we modelled and predicted human source credibility evaluations using EEG brain activity measurements with F1 score exceeding 0.7 (using 10-fold cross-validation).
\end{abstract}

\section{Introduction}

Fake news, or false news, has become a buzz-word to describe a general problem that emerged when the Web achieved critical mass in developed societies. In 2016, Web-based social media became a source of news for over $60 \%$ of adult Americans [1]. This situation coincided with the American presidential election campaign, which revealed the Web's weakness: lack of mechanisms for Web content credibility evaluation. Google trends show that since then (October, 2016), the term fake news (which has been in use since 19th century) has rapidly grown in popularity ${ }^{1}$. Fake news are a subject of active research, but at the same time they are still poorly understood [14]. Web-based social media, such as Facebook or Twitter, are especially vulnerable to fake news proliferation [1], because messages on social media are forwarded based on the trust that receivers have in their virtual friends (or followers in Twitter sources). It is, therefore, crucial to research the evaluation of source credibility.

\footnotetext{
${ }^{1}$ Leetaru, Kalev. "Did Facebook's Mark Zuckerberg Coin The Phrase 'Fake News'?". Forbes.
}

(C) Springer Nature Switzerland AG 2020

V. V. Krzhizhanovskaya et al. (Eds.): ICCS 2020, LNCS 12137, pp. 327-341, 2020.

https://doi.org/10.1007/978-3-030-50371-0_24 
Social psychology has contributed to our understanding of social and psychological factors that affect credibility evaluations made by a information receiver $[7,21]$. However, all these findings are based on behavioral studies that rely on participant declarations or on indirect inferences from observations of participant behavior. Simply asking experiment participants whether they believe fake news (or inferring this information from observed behavior) may not reveal the real reasons for such a decision (some of these reasons may not even be consciously known by the experiment participants). By directly observing brain activity, researchers can understand basic processes that occur in the brain during credibility evaluations.

In turn, knowledge about brain activity during credibility evaluation could be applied by neuroinformatics in order to create an EEG-based measure of credibility. However, most research that used EEG or fMRI in the context of credibility has focused on lie detection [16,24], which is based on the investigation of the brain activity of the author, and not the receiver of the message.

\subsection{Research Problem and Contributions}

In order to study credibility empirically, it is useful to deconstruct this complex concept into simpler ones. This is the approach adopted in our research. In this article, we deal with one of such simpler concepts: source credibility. Because source credibility evaluation has not been studied using brain activity analysis before, our research goals are largely exploratory and we do not have preconceived expectations based on literature. The goal of this article is to address the following research questions:

- What brain areas, and in which time intervals since the stimulus, are active when a receiver makes positive or negative source credibility evaluations?

- How does brain activity depend on the level of source credibility?

- Can we model and predict human source credibility evaluations using EEG brain activity measurements?

While a comprehensive answer to the questions listed above would require additional studies, this article makes significant contributions to this matter. For the first time in literature, in this article, there are described areas of the brain (Brodmann areas) involved in the decision making process based on source credibility. Using this knowledge, this article describes an operational model of decision making based on source credibility that uses EEG measurements as an input. Not only does this model provide basic knowledge in the field of neuroinformatics, but it can be seen as a first step towards a practical EEG-based measurement method of source credibility.

In the next section, we introduce a definition of source credibility and discuss theoretical research that can guide the design of empirical experiments for studying credibility. We also discuss related work that studied brain activity related to source credibility evaluation. In Sect. 3, we describe the design of our experiment. Section 4 discusses the experiment results. Section 5 concludes the article and introduces our plans for future work. 


\section{Related Work}

\subsection{Source, Message, Media Credibility}

The concept of credibility, similarly to the concept of trust, is grounded in science and in common sense. Credibility has been subject to research by scientists, especially in the field of psychology and media science. One of the earliest theoretical works on credibility dates back to the 1950s. This influential work of the psychologist Carl Hovland [8] introduced the distinction between source, message, and media credibility. Out of these three, two are a good starting point for a top-down study of the complex concept of credibility: source credibility and message credibility. These two concepts are closely related to the natural-language definitions of the term "credibility". In the English language dictionary (Oxford Advanced Learner's Dictionary), credibility is defined as "the quality that somebody/something has that makes people believe or trust them". When this definition is applied to a person ("somebody"), it closely approximates source credibility - an essential concept in real-life, face-to-face communication. However, notice that the dictionary definition of credibility can also be applied to "something" - the message itself. In many online environments, message credibility must be evaluated without knowledge about the source.

Information scientists have studied credibility evaluations with the goal of designing systems that could evaluate Web content credibility automatically or support human experts in making credibility evaluations [15,25]. Credibility evaluations, especially of source credibility, are significant in online collaboration, for example on Wikipedia [23,27]. However, human credibility evaluations are often subjective, biased or otherwise unreliable [11,19], making it necessary to search for new methods of credibility evaluation, such as the EEG-based methods proposed in this article.

\section{$2.2 \quad$ Source Credibility}

A search for the term "source credibility" on Google Scholar returns an excess of 12,000 results (for an overview of recent publications, especially on the subject of Web content credibility, see [26]). Research on this subject has ranged from investigating impact of source credibility on politics [6] to healthcare [12].

Previous theoretical research hypothesized that source credibility is closely related to credibility trust [26]. Credibility trust is an expectation that the source will observe the social norm of not lying (not communicating a false message). Following the analogy to trust, source credibility can also be based on the trustworthiness of the source in the context of veracity; it is difficult, however, to reliably observe, measure or predict this property. Most observations or valuations concerning credibility are done in a relational setting: communication of a message from a source to a receiver. A proxy for credibility trustworthiness may be source reputation in the context of veracity, estimated based on the past performance of the source. Therefore, it can be concluded that source credibility is a combination (or multiple criteria evaluation) of two kinds of trust: credibility 
trust and the trust in the expertise of the source. These two types of trust are independent and complementary; a source may, after all, usually tell the truth, but not be able to do so because of lack of expertise in a given subject. On the other hand, an expert in the subject may not be trustworthy due to the fact of being a habitual liar.

\subsection{Research on Brain Activity Related to Source Credibility Evaluation}

Not much has been done in the field of source credibility research as far as neuroimaging methods are concerned. Source credibility is associated with trustworthiness which was discussed, for example, by [20]. They state that usually the amygdala is involved in trusting others [20]. However, some findings $[3,10]$ prove that insular cortex has function similar to orbitofrontal cortex and plays an important role in decision making process [20]. The Brodmann area BA47 is anatomically located in orbitofrontal cortex and its activity during the process of making risky decisions was observed in Positron Emission Tomography (PET) study reported in [5]. The BA46 was found to be involved in decision making process in the presence of fatiguing factors in magnetoencephalographic research (MEG) [9] as well as in the [4] where comparison of moral and cognitive judgments was conducted. Together with BA47 where patients with reduction of ventrolateral prefrontal cortex gray matter played an economic game with some degree of irrationality implemented [2]. All above-mentioned papers show (using various techniques like PET, MEG and fMRI) that decision making, game theory, trustworthiness and judgment tasks are related and involve the BA46 and BA47 Brodmann areas. However, no research so far has identified areas of the brain involved in source credibility evaluation.

To sum up the Dorsolateral Prefrontal Cortex (DLPFC) BA46 is reported to be engaged in working memory oriented tasks requiring cognitive effort while BA47 is involved in decision-making process, especially in morally difficult problems $[2-5,9,10,20]$. Those are potential candidates to observation with greatest attention in our experiment.

\section{Experiment Design}

We have designed and conducted a pilot experiment to study source credibility evaluations using EEG. The pilot experiment was carried out at Marie Curie Skłodowska University in Lublin, Poland, from June, 15th till July, 14th, 2019 (MCSU Bioethical Commission permission 13.06.2019).

The aim of the pilot experiment was to observe activity of the participant's brain cortex during performance of a task involving source credibility evaluation. In order to ensure that the participants could rely only on source credibility during the experiment, the experiment was designed so that the participants would not be familiar with the topics of the messages. The selected message topics concerned a Japanese language test. All experiment participants had no knowledge of Japanese. 
In order to simplify EEG measurement, all participants selected for the experiment were right-handed males. A total of 57 participants took part in the pilot experiment.

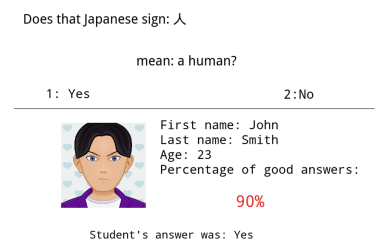

Fig. 1. Typical screen shown to participant during the experiment. The student's hint, accuracy during the test, and avatar are in the bottom section of the screen. The participant is asked to agree or disagree with the student's answer (top section) based on their trust or distrust in the hint that are influenced by the student's accuracy.

\subsection{Source Credibility Evaluation Task}

In the introduction to the experiment, participants were informed that students of another university had solved a test of their knowledge of Japanese Kanji signs (after one semester of learning completed) and that we know the results achieved by all students.

To the end of our experiment, randomly generated names of students were chosen together with avatars representing their faces. Avatars were generated by means of repository available in www.makeavatar.com and a simple Python script. The neutral emotions were provided by the smile-option turned off. The 'generated' students fell into three groups - those who received 50\%, 70\% and $90 \%$ of the maximum score to be gained during the test.

Participants were shown 180 screens with one Kanji sign on each of them and the question if the translation of that sign was correct or not. As a hint, participants received information about students (represented by name and avatar) who had an overall accuracy of $50 \%, 70 \%$ or $90 \%$ during the test. The hint was the student's answer ('Yes' or 'No') to the question posed to the participant (see Fig. 1). The same accuracy of the hinting student was shown on 60 screens (with values $50 \%, 70 \%$ or $90 \%$ ).

Note that the participants did not know whether the student's response was correct or incorrect. The only thing a participant knew was the student's result in the entire test. In this way, we have created a situation in which the participant had to make a decision whether to accept a message (the student's hint) based on source credibility (the student's overall score in the test).

In the experiment, the participants faced a binary decision: they were asked to press a 'YES' or 'NO' button. This decision could comply with the student's hint, in which case we shall refer to this decision as "trusting". The participants could also disagree with the student's hint, in which case we shall speak of "distrust". Note that both trusting and distrusting decisions can be 'YES' or 
'NO', but this is not relevant for the experiment. The only relevant aspect of the participant's decision is whether it is trusting or distrusting, corresponding to a positive or negative source credibility evaluation, respectively.

Recall that source credibility can be measured as the source's reputation in the context of expertise or veracity. In our experiment, the only information that participants had about the students' reputation was the test score. If the participants were informed that the suggesting student's test score was $90 \%$, they would probably make a trusting decision. If the test score was $50 \%$, we could expect that the participant would respond randomly. The most interesting situation was when the test score was $70 \%$. We shall refer to the hinting student's test score as the Source Credibility Level (SCL).

Moreover, the participants were not in any case given the correct meaning of the current and previous signs presented to them. Thus, the participants were not rewarded for a good answer and were not punished for a bad one. This experiment design ensured that the participant made decisions in a non-competitive setting and without consideration for a reward.

Repeating similar screens 60 times for each source credibility level made it possible to observe the so-called Event-Related Potentials (ERPs) in the electroencephalographic activity registered by the amplifier in our lab. The methodology of ERP is probably most often used in experimental psychology and observations made using source localisation methods allowed us to measure brain cortex activity quantitatively.

\subsection{Experimental Cases and Data}

All decisions made by experiment participants can be classified into the six following cases that allow us to compare brain activity for trusting and distrusting decisions under stimulus of various source credibility levels:

- T50: Source credibility was $50 \%$ and the subject trusted the message.

- D50: Source credibility was 50\% and the subject did not trust the message.

- T70: Source credibility was $70 \%$ and the subject trusted the message.

- D70: Source credibility was $70 \%$ and the subject did not trust the message.

- T90: Source credibility was $90 \%$ and the subject trusted the message.

- D90: Source credibility was $90 \%$ and the subject did not trust the message.

Additionally, let us introduce three larger sets of all decisions made while the participant was shown a particular source credibility level: $A 50=T 50 \cup D 50$, $A 70=T 70 \cup D 70$ and $A 90=T 90 \cup D 90$.

\subsection{Experiment Hypotheses}

The experiment was designed to study the three research questions described in the introduction. We observed and analysed participants' brain activity during the source credibility evaluation task. The experiment's design had limited the stimulus received by participants to source credibility, and the participants had 
to make a binary decision. Our pre-hypothesis was, therefore, that there existed a positive relationship between source credibility and average number of trusting decisions.

The next hypotheses concern participants' brain activity: specifically, the average amplitudes of ERP signals measured by cognitive processing electrodes in cognitive processing time interval (450-580 $\mathrm{ms}$ from stimuli) that will be referred as CPTI. For short, we shall refer to these ERP signals as cognitive ERP signals. We make the following hypotheses related to the first two research questions concerning brain activity for trusting and distrusting decisions on the basis of source credibility:

1. the ERP signals from all 26 cognitive electrodes in a certain time interval have statistically significant differences for different source credibility levels of $50 \%, 70 \%$ and $90 \%$ (in cases A50, A70, A90);

2. the ERP signals from all 26 cognitive electrodes in a certain time interval have statistically significant differences for pairs of cases: T50 and T90, D50 and D90, T70 and T90, D70 and D90;

3. the ERP signals from all 26 cognitive electrodes in a certain time interval have statistically significant differences for cases: T90 and D90, T70 and D70, T50 and D50;

When verifying hypotheses 1, 2, and 3, we will investigate time intervals within the cognitive decision making time interval and select the longest time interval during which a hypothesis holds. The comparison of these time intervals for the various hypotheses brings additional insight into the analysis.

The next hypothesis concerns the mean electric charge (MEC) flowing through all Brodmann Areas (BAs). We have used these measurements to consider the third research question: whether it is possible to model and predict source credibility evaluations using EEG measurements.

5. the mean electric charge flowing through estimated Brodmann Areas (BA) is sufficient to predict the decision to trust or distrust during the experiment, with an accuracy that significantly exceeds the baseline.

Note that hypothesis 5 concerns the possibility of creating an EEG-based method of source credibility measurement. While this is only a first step, a positive validation of hypothesis 5 would open an avenue of investigating EEG-based source credibility measurement in other, more complex and realistic scenarios. Note that the baseline accuracy for hypothesis 5 is $50 \%$ (experiment participants make binary decisions).

\subsection{EEG Measurements}

Our empirical experiments involved top EEG devices. We were equipped with a dense array amplifier recording the cortical activity with up to $500 \mathrm{~Hz}$ frequency 


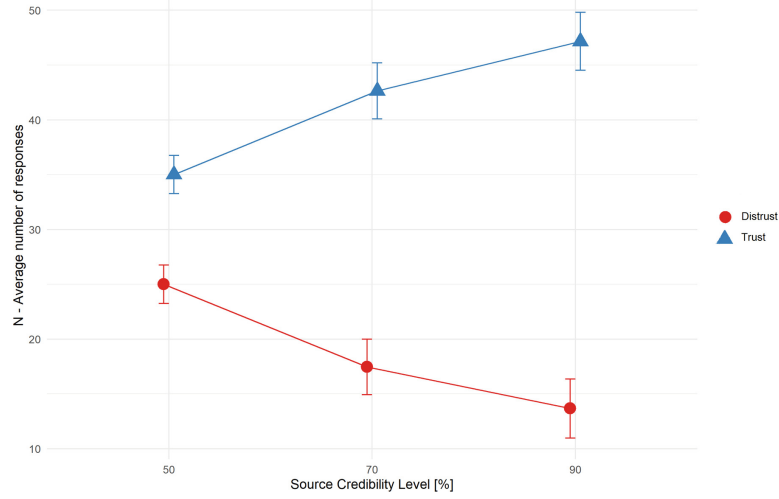

Fig. 2. Average number of trusting and distrusting responses given by participants when evaluating different source credibility levels $50 \%, 70 \%$ and $90 \%$. The increase in trust and decrease in distrust can be observed with source credibility level increase.

through 256 channels HydroCel GSN 130 Geodesic Sensor Nets provided by $\mathrm{EGI}^{2}$. In addition, in the EEG Laboratory the Geodesic Photogrammetry System (GPS) was used.

Estimating ERP for each of the 256 electrodes is not necessary for ERP observation, as in general standards there are just a few electrodes (in our case 26) playing an important role in cognitive tasks. ${ }^{3}$ However, for the sLORETA source localisation analyses (used for verification of the next hypotheses) the ERP for all 256 electrodes had to be in fact calculated on the fly. Therefore, in the beginning the raw EEG time series were post-processed, averaged and ERPs were estimated from 26 cognitive electrodes $[13,17,22]$. In the following discussion, when we refer to differences in the cognitive ERP signal, it means that in a certain time interval the average ERP signal from all 26 cognitive electrodes was different.

Having the ERP signal estimated for each electrode out of 256, it was possible to calculate the mean electric charge (MEC) flowing through the BA situated under these electrodes on the brain cortex in CPTR. Moreover, it was also possible to conduct the full source localisation analysis of the signal originating from all 256 electrodes using sLORETA algorithm (GeoSourse parameters set as follow: Dipole Set: 2 mm Atlas Man, Dense: 2447 dipoles Source Montages: BAs). Mean electric current flowing through each BA and varying in time was given as

${ }^{2}$ Electrical Geodesic Systems, Inc., 500 East 4th Ave. Suite 200, Eugene, OR 97401, USA.

3 The electrodes are described in EGI 256-channel cap specification as best for cognitive ERP observations, covering the scalp regularly and numbered as follows: E98, E99, E100, E101, E108, E109, E110, E116, E117, E118, E119, E124, E125, E126, E127, E128, E129, E137, E138, E139, E140, E141, E149, E150, E151, E152. Those electrodes are automatically chosen for observing P-300 ERP signal by NetStation software. 

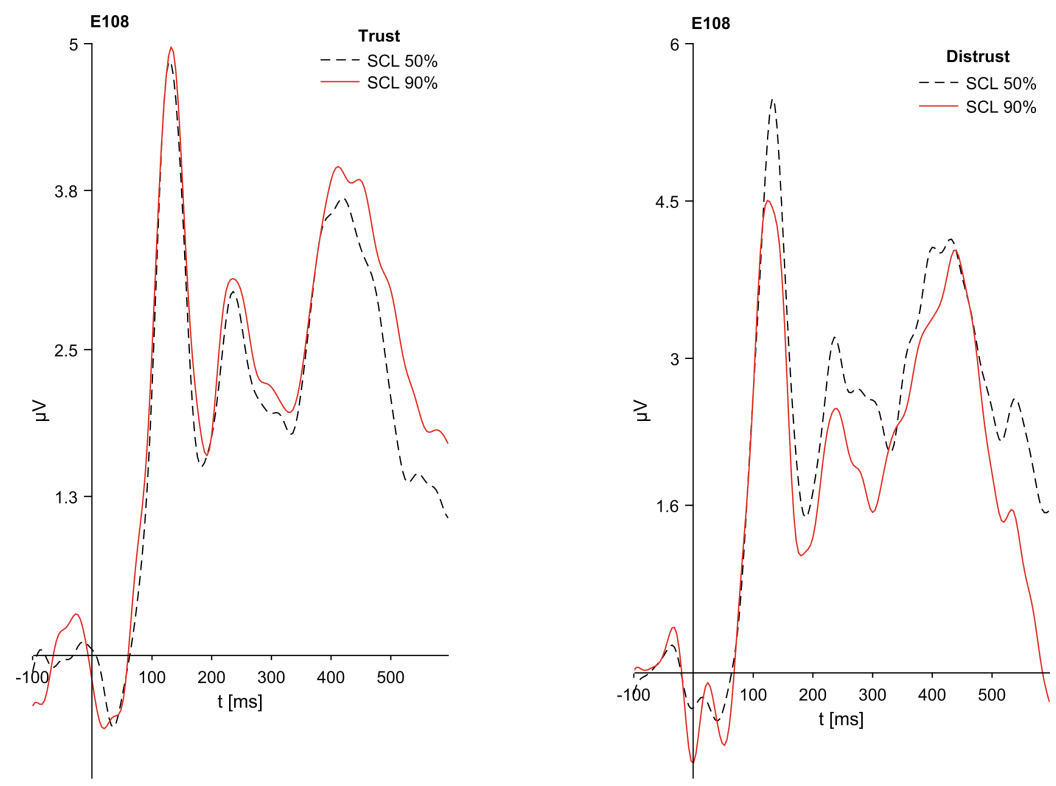

Fig. 3. The ERP plots averaged for all participants from $-100 \mathrm{~ms}$ before stimuli to $600 \mathrm{~ms}$ after that for source credibility levels $50 \%$ and $90 \%$. Results obtained for one electrode No. 108. The statistical difference in cortical activity is present both for the T50 \& T90 (in the left) and distrust D50 \& D90 (in the right) responses.

an output. Having those values calculated, it was possible to integrate that current in time and then get the MEC. The mean electric charge calculated for each electrode using source localisation techniques could, as we intended, indicate the hyperactivity of some BAs that are not necessary precisely situated under the cognitive electrodes. For all calculations of MEC the CPTR was divided into $10 \mathrm{~ms}$ time intervals. The procedure of calculating MEC has been described in detail in [29].

We shall denote the MEC by $\mu_{b}^{\left(t_{1}, t_{2}\right)}$, where $b$ is the index of the Brodmann area, while $\left(t_{1}, t_{2}\right)$ is the time interval. Note that $t_{2}-t_{1} \geq 10 \mathrm{~ms}$, but we can also calculate the MEC in longer time intervals. Note also that we calculate the MEC based on ERP signals calculated from a subset of participant decisions usually from a subset of identical decisions (to trust or to distrust the source). Therefore, the variables $\mu_{b}^{\left(t_{1}, t_{2}\right)}$ can be used as independent variables related to a single participant's decision.

\section{Experiment Results}

As far as the pre-hypothesis is concerned, it was indeed possible to observe a relationship between subjects' responses and Source Credibility Level (SCL) during the experiment. When the SCL was set to $50 \%$, on average 35 out of 60 
suggestions of correct word meaning were trusted and 25 were not. Similarly, for the SCL equal to $70 \%$, on average 42.64 suggestions were trusted and 17.47 were not. In the case of SCL $=90 \%$, the average of 44.52 suggestions were trusted and 10.98 were not. For details see Fig. 2. This observation demonstrates that the pilot experiment has a high internal validity.

\subsection{Brain Activity Measurements}

For the verification of hypotheses 1 the Pearson's chi-squared test with 2 degrees of freedom was used with a $p$ value of 0.05 . Hypothesis 1 has been confirmed. For all 3 levels of SCL (regardless of the decision made by participants), a statistically significant difference in the cognitive ERP signal was observed. These differences manifest between $340 \mathrm{~ms}$ and $540 \mathrm{~ms}$ after showing the stimulus.

A statistically significant difference in the ERP signals from all 26 cognitive electrodes for the comparison of SCL $=50 \%$ and SCL $=90 \%$ manifests itself in time range from $260-600 \mathrm{~ms}$ in both cases when subjects trust (comparison of T50 and T90) and do not trust (D50 and D90). See Fig. 3. A statistically significant difference can be also observed for ERP signals on all 26 cognitive electrodes between SCL $=70 \%$ and SCL $=90 \%$ for trusting decisions (comparison of T70 and T90) cover the entire CPTI. It is interesting that there is no statistically significant difference in case of distrusting decisions (comparison of D70 and D90). This means that the hypothesis 2 is partially confirmed.

As far as the hypothesis 3 is concerned, it was also possible to find statistically significant differences for certain time intervals when comparing trusting and distrusting ERPs for all three SCLs. The ERP signal collected from cognitive electrodes was significantly different between $360 \mathrm{~ms}$ and $600 \mathrm{~ms}$ when the subject was responding at SCL $=70 \%$ (comparison of T70 \& D70). For SCL $=50 \%$, statistically significant differences could be observed in 390-460 ms and 500-560 ms (comparison of T50 \& D50), while for SCL $=90 \%$ the ERP was significantly different in the interval of $480-530 \mathrm{~ms}$ and $550-600 \mathrm{~ms}$ since the stimulus (comparison of T90 \& D90).

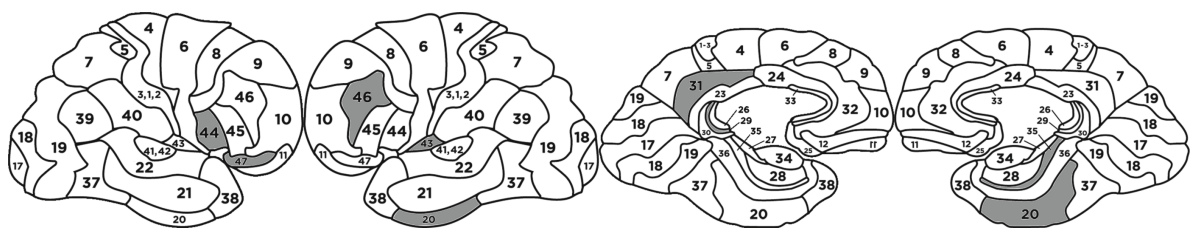

Fig. 4. Brodmann areas most significant for predicting source credibility evaluations 
Table 1. Brodmann Areas that manifest statistically significant differences in the spectrum of MEC for trust and distrust decisions during source credibility evaluation. Presented with their corresponding anatomical structure of the brain and known functions as listed in [18]. The prefix of particular BAs stands for L-eft and R-ight hemispheres.

\begin{tabular}{l|l|l}
\hline BA & Anatomical structure & Known functions \\
\hline L-BA46 & Dorsolateral Prefrontal Cortex & $\begin{array}{l}\text { Memory encoding and recognition internal } \\
\text { mental calculation processing emotions } \\
\text { self-reflections in decision making }\end{array}$ \\
\hline R-BA29 & Granular retrosplenial cortex & Related to language memory retrieval \\
\hline L-BA20 & Inferior Temporal Gyrus & $\begin{array}{l}\text { Lexico-semantic processing language } \\
\text { comprehension and production }\end{array}$ \\
\hline L-BA35 & Perirhinal area & Memory encoding \\
\hline L-BA43 & Primary Gustatory Cortex & Language comprehension \\
\hline R-BA47 & Inferior Prefrontal Gyrus & $\begin{array}{l}\text { Decision making working memory semantic } \\
\text { encoding active semantic retrieval } \\
\text { phonological expression single word reading }\end{array}$ \\
\hline R-BA44 & Pars Opercularis & $\begin{array}{l}\text { Working memory expression of emotional } \\
\text { information sentence comprehension word } \\
\text { and face encoding Solving arithmetical tasks }\end{array}$ \\
\hline R-BA31 & Dorsal posterior cingulate & $\begin{array}{l}\text { Emotion related to language attention to } \\
\text { speech }\end{array}$ \\
\hline
\end{tabular}

\subsection{Regression Model of Source Credibility Evaluations}

We divided participant responses into two equal parts. The first part consisted of cognitive ERPs calculated on the basis of first 90 questions (30 for every level of SCL), and the second part consisted of cognitive ERPs calculated on the questions 91-180. Next, the MECs were calculated for both parts. The first part was used to create the training set, while the second part to create a validation set for the models of the participants' brain processes and decision making.

The explanatory (independent) variables of the model were MECs based on the ERPs of participants. Recall that we have denoted MEC by $\mu_{b}^{\left(t_{1}, t_{2}\right)}$, where $b$ is the index of the Brodmann area, while $\left(t_{1}, t_{2}\right)$ is the time interval. The model is based on MECs of all participants. These MECs are calculated in the time interval that had the highest differences of ERP for all Brodmann areas. The independent variables of the model are $x_{b}^{p}=\mu_{b}^{(150 \mathrm{~ms}, 600 \mathrm{~ms})}$. The training set of the model is 68 observations (the number of participants) of each class (136 observations in total), and the validations set has the same size.

The number of explanatory variables was reduced by considering a subset of variables that had the largest Wald statistic in the first model with all variables.

We created a universal Backward Stepwise Logistic Regression with Wald criterion classifier in SPSS. We choose generalized logistic regression in order to gain higher insight into the brain activities during source credibility evaluation (using other classifiers could increase classification accuracy). 
The classifier achieved satisfactory characteristics in 14 steps with Nagelkerke's $R^{2}=0.38$. The Brodmann areas L-BA46, R-BA29, L-BA20 and L-BA35 (see first four rows of Table 1) had the highest impact on the classification with regression parameters $\beta$ equal to 180.24 , 146.17, 126.30, 90.87 for each mentioned above BA respectively. The most significant Brodmann areas selected by the classifier are also shown on anatomical maps of the brain in Fig. 4.

We have used 10-fold cross-validation to confirm our results and the average efficiency achieved by classifiers in this cross-validation are as follows: Accuracy $=0.695$, Precision $=0.667$, Recall $=0.781$ and F1score $=0.719$ which proves the stability of our results.

An F1 accuracy of $70 \%$ with a baseline of $50 \%$ is sufficiently high to consider that hypothesis 5 holds.

\subsection{Discussion and Limitations}

The results of our analysis concerning the first two research questions: "What activities occur in the brain when a receiver is evaluating source credibility?" and "How does brain activity depend on the level of source credibility?" revealed differences in brain activity on all cognitive electrodes during time intervals of at least $100 \mathrm{~ms}$. The statistically significant difference in the EEG activity for different source credibility levels manifests both in the ERP curves' shapes generated for the cognitive electrodes, and in the mean electric charge flowing through particular Brodmann Areas. The mean electric charge approach was proposed in [29] and seems to work in the full spectrum of qEEG analysis [28,30].

Differences are observed in the interval of time in which the cognitive processing takes place. As the SCL had a strong influence on the decisions made by participants we can believe that it was methodologically correct. In most cases, the ERP signals significantly differ both for the trust and distrust decisions and for the particular SCLs.

When analysing the mean electric charge, the most significant differences in brain activity were found in the Dorsolateral Prefrontal Cortex, granular retrosplenial cortex, Inferior Temporal Gyrus and perirhinal area. These brain modules are responsible for a variety of functions (see Table 1), including working memory, decision making and lexical-semantic processing. The observations reported in this article are new results and point out new functions of these brain areas: the function of source credibility evaluation.

It is worth noting, however, that the word and face encoding areas, as well as the unit responsible for solving mathematical tasks (Pars Opercularis), were also engaged (although they had weaker impact on decisions). This can lead to the conclusion that during the source credibility tasks, some probabilistic calculations are conducted and the face of the information sender may also play an important role in the decision making process. However, these observations may also be a consequence of our experiment design.

Note, that BA47 and BA46 reported by us as manifesting statistically significant difference in activity and playing such an important role in our model were also reported in similar tasks involving decision-making about trustworthiness and judgements $[2-5,9,10,20]$. 
Our results concerning the other two research questions: "Can we model and predict human source credibility evaluations using EEG brain activity measurements?" and "Can we create a source credibility measurement method based on EEG brain activity measurements?" are promising. We showed that using EEG methods, we can model and predict human source credibility evaluations.

A model based on logistic regression was proposed for classifying the trusting or distrusting decisions of experiment participants. The accuracy of the models is high compared to the baseline. This shows that our model can be considered a first step towards source credibility measurement method based on EEG. Such a method would have wide applications, for example the measurement of source credibility of election candidates or journalists. However, these applications would have to be tested using follow-up experiments in the future.

\section{Conclusion and Future Work}

In future work, we intend to test other classifiers to check their accuracy in the discussed classification tasks. Some parameter search simulations will be performed as well, and we hope that it is possible to find the set of Brodmann Areas characteristic not only for the entire cohort, but also for individual brains.

On the other hand, increasing the number of participants should let us build a universal model classifying brain cortical dynamics with a high level of accuracy. It is justified to hypothesise that while individual brains are different, when we evaluate more participants, there is a higher chance of finding similar brains.

Our investigation studied source credibility, which is a single aspect of credibility evaluation. Our research will continue with the investigation of message credibility, as well as a holistic credibility evaluation. We hope that our research will one day lead to proposing a comprehensive, quantitative model of the credibility phenomenon, based on electrical brain activity.

\section{References}

1. Allcott, H., Gentzkow, M.: Social media and fake news in the 2016 election. J. Econ. Perspect. 31(2), 211-236 (2017)

2. Chung, H.K., Tymula, A., Glimcher, P.: The reduction of ventrolateral prefrontal cortex gray matter volume correlates with loss of economic rationality in aging. J. Neurosci. 37(49), 12068-12077 (2017)

3. Clark, L., Bechara, A., Damasio, H., Aitken, M., Sahakian, B., Robbins, T.: Differential effects of insular and ventromedial prefrontal cortex lesions on risky decisionmaking. Brain 131(5), 1311-1322 (2008)

4. Deppe, M., et al.: Nonlinear responses within the medial prefrontal cortex reveal when specific implicit information influences economic decision making. J. Neuroimaging 15(2), 171-182 (2015)

5. Ernst, M., et al.: Decision-making in a risk-taking task: a pet study. Neuropsychopharmacology 26(5), 682-691 (2002)

6. Flanagin, A.J., Metzger, M.J.: Digital media and perceptions of sourcecredibility in political communication. Oxf. Handb. Polit. Commun. 417, 1 (2017) 
7. Forgas, J.P., Baumeister, R.: The Social Psychology of Gullibility: Conspiracy Theories, Fake News and Irrational Beliefs. Routledge, Abingdon (2019)

8. Hovland, C.I., Weiss, W.: The influence of source credibility on communication effectiveness. Public Opin. Q. 15(4), 635-650 (1951)

9. Ishii, A., Tanaka, M., Watanabe, Y.: The neural mechanisms underlying the decision to rest in the presence of fatigue: a magnetoencephalography study. PLoS ONE 9(10), e109740 (2014)

10. Jones, C.L., Ward, J., Critchley, H.D.: The neuropsychological impact of insular cortex lesions. J. Neurol. Neurosurg. Psychiatry 81(6), 611-618 (2010)

11. Kakol, M., Jankowski-Lorek, M., Abramczuk, K., Wierzbicki, A., Catasta, M.: On the subjectivity and bias of web content credibility evaluations. In: Proceedings of the 22nd International Conference on World Wide Web, pp. 1131-1136. ACM (2013)

12. Kareklas, I., Muehling, D.D., Weber, T.: Reexamining health messages in the digital age: a fresh look at source credibility effects. J. Advert. 44(2), 88-104 (2015)

13. Kawala-Sterniuk, A., et al.: Comparison of smoothing filters in analysis of EEG data for the medical diagnostics purposes. Sensors 20(3), 807 (2020)

14. Lazer, D.M., et al.: The science of fake news. Science 359(6380), 1094-1096 (2018)

15. Liu, X., Nielek, R., Adamska, P., Wierzbicki, A., Aberer, K.: Towards a highly effective and robust web credibility evaluation system. Decis. Support. Syst. 79, 99-108 (2015)

16. Meijer, E.H., Verschuere, B.: Deception detection based on neuroimaging: better than the polygraph? J. Forensic Radiol. Imaging 8, 17-21 (2017)

17. Mikołajewska, E., Mikołajewski, D.: Ethical considerations in the use of braincomputer interfaces. Open Med. 8(6), 720-724 (2013)

18. Pascual-Leone, A., Davey, N.J., Rothwell, J., Wasserman, E.M., Puri, B.K.: Handbook of Transcranial Magnetic Stimulation, vol. 15. Arnold, London (2002)

19. Rafalak, M., Abramczuk, K., Wierzbicki, A.: Incredible: is (almost) all web content trustworthy? Analysis of psychological factors related to website credibility evaluation. In: Proceedings of the 23rd International Conference on World Wide Web, pp. 1117-1122. ACM (2014)

20. Rosenbloom, M.H., Schmahmann, J.D., Price, B.H.: The functional neuroanatomy of decision-making. J. Neuropsychiatry Clin. Neurosci. 24(3), 266-277 (2012)

21. Rutjens, B.D., Brandt, B.D.: Belief Systems and the Perception of Reality. Routledge, Amsterdam (2018)

22. Tadeusiewicz, R., et al.: Neurocybernetyka teoretyczna. Wydawnictwa Uniwersytetu Warszawskiego, Warsaw (2009)

23. Turek, P., Wierzbicki, A., Nielek, R., Datta, A.: Wikiteams: how do they achieve success? IEEE Potentials 30(5), 15-20 (2011)

24. Wang, H., Chang, W., Zhang, C.: Functional brain network and multichannel analysis for the P300-based brain computer interface system of lying detection. Expert. Syst. Appl. 53, 117-128 (2016)

25. Wawer, A., Nielek, R., Wierzbicki, A.: Predicting webpage credibility using linguistic features. In: Proceedings of the 23rd International Conference on World Wide Web, pp. 1135-1140. ACM (2014)

26. Wierzbicki, A.: Web Content Credibility. Springer, Cham (2018). https://doi.org/ 10.1007/978-3-319-77794-8

27. Wierzbicki, A., Turek, P., Nielek, R.: Learning about team collaboration from Wikipedia edit history. In: Proceedings of the 6th International Symposium on Wikis and Open Collaboration, pp. 1-2 (2010) 
28. Wojcik, G.M., et al.: Mapping the human brain in frequency band analysis ofbrain cortex electroencephalographic activity for selected psychiatricdisorders. Front. Neuroinformatics 12, 73 (2018)

29. Wojcik, G.M., et al.: New protocol for quantitative analysis of braincortex electroencephalographic activity in patients with psychiatricdisorders. Front. Neuroinformatics 12, 27 (2018)

30. Wojcik, G.M., et al.: Analysis of decision-making process using methods of quantitative electroencephalography and machine learning tools. Front. Neuroinformatics 13, $73(2019)$ 